\title{
Pelvis motion characteristics in case of induced gait perturbation in male and female adults
}

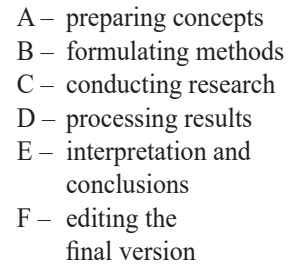

Received: 2019-07-15 Accepted: 2019-08-05

\author{
Barbara Łysoń ${ }^{1, A-D}$, Katarzyna Bienias ${ }^{1, A-B, E}$, Joanna Ścibek ${ }^{1,2, A-B, E}$, \\ Andrzej Wit ${ }^{1, A, E-F}$
}

${ }^{1}$ Faculty of Rehabilitation, The Jozef Pilsudski University of Physical Education in Warsaw

${ }^{2}$ Department of Physiotherapy, College of Rehabilitation in Warsaw

https://doi.org/10.5114/areh.2019.87746

\section{Key words: stability, pelvis, perturbation, fall prevention}

\section{Introduction}

Falls are a serious social problem as well as a difficult medical challenge, especially in the elderly. From 31 up to $38 \%$ of people over 65 years old fall every year [1-3] and this number increases with age $[2,3]$. Moreover, falls often occur while walking $[1,4]$. Falls may result in serious injuries such as the fracture of the neck of the femur, which requires medical care and long immobilization. On the other hand, falls may lead to the post-fall syndrome, which is the fear of falling again that substantially decreases the level of activity in older people.

Falls also regularly occur in younger adults and are the main reason for workplace injuries [5]. The expenditures related to the treatment as well the insurance come down to around $15 \%$ of the costs 
related to all injuries, what in the United States amounts to 19 billion dollars annually [2].

The factors increasing the risk of falling may be divided into two groups: internal and external ones. Internal risk factors are related to decreased physical fitness, for example, reduced muscle force, eye disorders or cognitive functions disorders. External risk factors (also called environmental risk factors), that add up to over $1 / 3$ of falls [6,7], include among others slippery or uneven surface, poor lighting, or inappropriate footwear [8].

Another internal factor that can increase the risk of falling is gender. Authors reported, that women fall around 1.2 times more frequentlycompared to men $[2,4]$, while for people whose age exceeded 85 years old this number reached 1.9 [2].

Sunget et al. [9] suggested that while evaluating the effect of perturbation, gender should be considered because of anatomical differences between males and females in the regions of lumbar spine and pelvis. The female pelvis is wider and shorter with the pelvis bone thinner and more delicate [10]. Additionally, in women pelvic tilt is greater than in men.
Pelvis kinematics and the differences between the sexes while executing different movement patterns were the subject of many studies and it was found that women exhibit greater pelvis tilt $[11,12]$ and greater range of motion in the coronal plane while walking compared to men [12].

The aim of this study was to compare differences between the selected values describing the pelvis motion while gait perturbation and the values recorded while normal walking, taking gender into consideration.

\section{Materials and methods}

The study group included forty-three adults (26 females and 16 males). Table 1 shows the group's characteristics together with the p-value for ShapiroWilk test and T-Student or U Mann-Whitney tests. Menand women were in similar age $(p=0.88)$, but differed in height $(\mathrm{p}<0.001)$ and body mass $(\mathrm{p}<0.001)$. Exclusion criteria included:lower limb or head injuries sustained within the last six months, diseases of the vestibular system, or serious eye disorders.

Tab. 1. Characteristic of the measured group together with p-value for Shapiro-Wilk test for females $\left(\mathrm{p}_{\mathrm{F}}(\mathrm{S}-\mathrm{W})\right)$ and males $\left(\mathrm{p}_{\mathrm{M}}(\mathrm{S}-\mathrm{W})\right)$ and $\mathrm{p}$-value $(\mathrm{p})$ for T-Student test $(\mathrm{T})$ or U Mann-Whitney $(\mathrm{U})$ test

\begin{tabular}{|c|c|c|c|c|c|c|}
\hline & Female & Male & Total & $P_{F}(S-W)$ & $\mathrm{p}_{\mathrm{M}}(\mathrm{S}-\mathrm{W})$ & $\mathrm{p}(\mathrm{U} / \mathrm{T})$ \\
\hline $\mathrm{N}$ & 27 & 16 & 43 & & & \\
\hline $\begin{array}{l}\text { Age [years] } \\
\text { mean } \pm \text { std }\end{array}$ & $23 \pm 4$ & $23 \pm 4$ & $23 \pm 4$ & $<0.001$ & $<0.001$ & $0.88(\mathrm{U})$ \\
\hline $\begin{array}{c}\text { Height }[\mathrm{m}] \\
\text { mean } \pm \text { std }\end{array}$ & $1.67 \pm 0.08$ & $1.81 \pm 0.07$ & $1.71 \pm 0.10$ & 0.648 & 0.745 & $<0.001(\mathrm{~T})$ \\
\hline $\begin{array}{l}\text { Body mass }[\mathrm{kg}] \\
\quad \text { mean } \pm \text { std }\end{array}$ & $62 \pm 10$ & $86 \pm 18$ & $71 \pm 18$ & 0.048 & 0.006 & $<0.001(\mathrm{U})$ \\
\hline
\end{tabular}

The research was approved by the local ethics committee (SKE 01-55/201.7). Additionally, the study was registered in the Clinical Trials database (NCT03813004).

Measurements were taken with the use of Motek Grail system equipped with 9 Vicon Bonita cameras, 3 video cameras, virtual reality component, and two-belts treadmill with inbuilt 6 degrees of freedom force plates. First, the informed consent to participate in the study was submitted by each subject, then anthropometric measurements were taken. Next, 25 reflective markers were placed on the subject's body according to the HBMmodel (Human Body Model) [13]. Subsequently, each participant was informed about the measurement procedure and secured with a safety harness.

\section{Measurement procedure:}

1. Familiarizing with the system's components, especially the treadmill, and adjusting the individual walking velocity ( 5 minutes)

2. Start of the measurement ( 60 seconds, normal gait)

3. Gait perturbation induced by the deceleration of the left treadmill belt (level 2 on a 5-level scale)

4. Completion of the measurements' recording, stopping the treadmill

Induced gait perturbation simulated a trip. The level 2 is characterized by the decrease of the treadmill belt velocity by $0.6 \mathrm{~m} / \mathrm{s}$ with the acceleration of $100 \mathrm{~m} / \mathrm{s}^{2}$, maintaining newly obtained speed for $0.3 \mathrm{~s}$ to finally return to the velocity from before the perturbation at the same acceleration rate. 
After the above described procedures were completed, the body composition analysis was conducted with the use of a TANITA monitor.

Values describing pelvis kinematics were the subject of the analysis. For rotation in the sagittal plane (pelvic tilt) forward tilt is positive, in the coronal plane (pelvic obliquity) left drop and the right lift is positive and in the transversal plane (pelvis rotation) left twist is positive.

The data were analyzed for left leg stance phase at the moment when the gait perturbation occurred and for the following stance phase of the contralateral leg. Fig. 1 presents a person who has been thrown off the balance during gait. Maximum and minimum rotation and obliquity values of the pelvis for left leg stance phase and maximum and minimum value of the pelvis tilt for the total analyzed time was calculated as well. Data analysis was conducted using Matlab software.

Statistical analysis was carried out with the STATISTICA 13 software using two-way repeatedmeasures analysis of variance with Tukey post-hoc test for different sample sizes. Repeated measures factor was called „PERT” and referred to perturbed and normal gait, while gender was selected to be the categorical factor. Additionally, assumptions of normality were checked with the use of ShapiroWilk and Levene test. The relationship between fat and muscle percentage, and analyzed parameters of the pelvis kinematics was examined. The assessment of this relationship was done using the Pearson correlation coefficient and scatter plots.

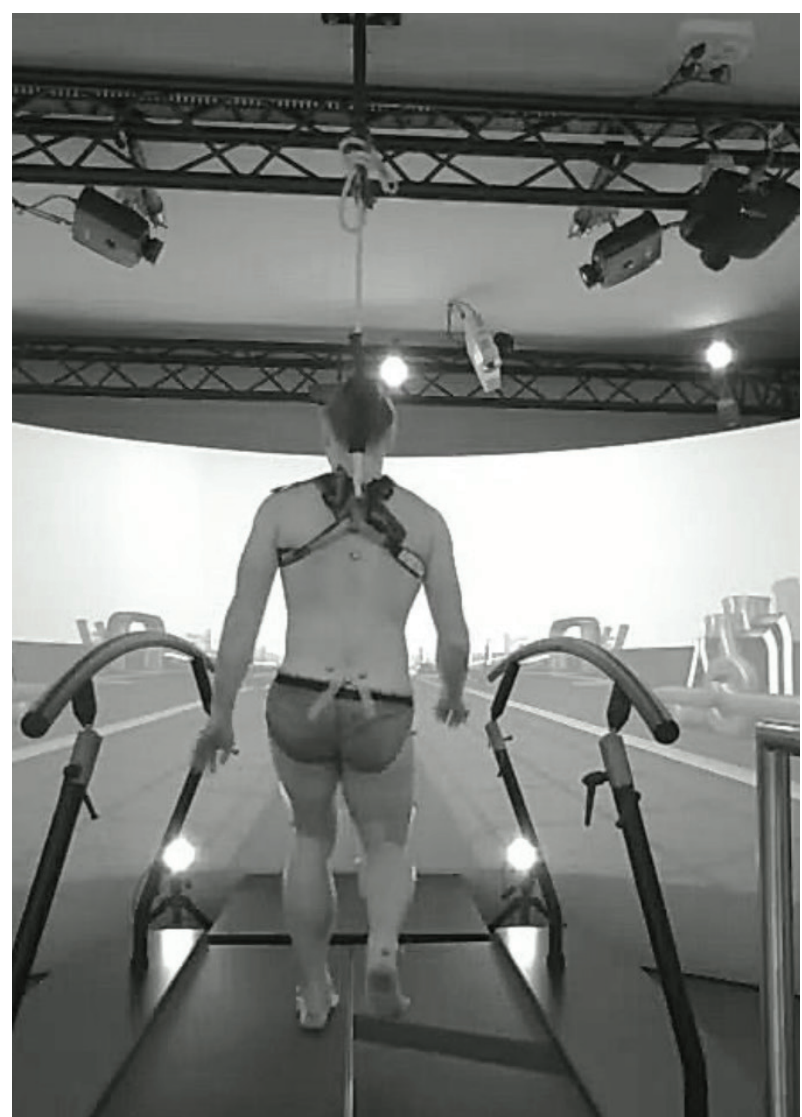

Fig. 1. Subjects reaction to gait perturbation

\section{Results}

Statistically significant differences between gait and perturbation were observed for each of the analyzed parameters, except for maximum rotation (Tab. 2).

Tab. 2 Results of the two-way analysis of variance (* stands for results for interaction)

\begin{tabular}{|c|c|c|c|c|}
\hline & Effect & $\eta^{2}$ & $\mathrm{~F}_{(1,41)}$ & $\mathrm{p}$ \\
\hline & Gender & 0.014 & 0.57 & 0.456 \\
\hline \multirow[t]{3}{*}{ obliquity $\min$} & PERT & 0.628 & 67.48 & $<0.001$ \\
\hline & PERT*Gender & 0.020 & 0.83 & 0.367 \\
\hline & Gender & 0.146 & $\mathbf{7 7 . 5 0}$ & 0.014 \\
\hline \multirow[t]{3}{*}{ obliquity max } & PERT & 0.553 & 48.29 & $<0.001$ \\
\hline & PERT*Gender & 0.110 & 4.84 & 0.034 \\
\hline & Gender & 0.221 & 10.76 & 0.002 \\
\hline \multirow[t]{3}{*}{ tilt $\min$} & PERT & 0.566 & 49.64 & $<0.001$ \\
\hline & PERT*Gender & 0.003 & 0.12 & 0.729 \\
\hline & Gender & 0.256 & 12.70 & 0.001 \\
\hline \multirow[t]{3}{*}{ tilt $\max$} & PERT & 0.659 & 71.56 & $<0.001$ \\
\hline & PERT*Gender & 0.025 & 0.94 & 0.339 \\
\hline & Gender & 0.276 & 15.28 & $<0.001$ \\
\hline \multirow[t]{3}{*}{ rotation $\min$} & PERT & 0.136 & 6.31 & 0.016 \\
\hline & PERT*Gender & 0.049 & 2.05 & 0.160 \\
\hline & Gender & 0.072 & 43.09 & 0.0864 \\
\hline \multirow[t]{2}{*}{ rotation $\max$} & PERT & 0.065 & 2.78 & 0.103 \\
\hline & PERT*Gender & 0.026 & 1.08 & 0.305 \\
\hline
\end{tabular}

Statistically significant value for $\alpha=0.05$ are bold. $\eta^{2}$ - partial eta squared, $F_{(1,41)}$ - test statistics value, $p$ - probability value 
In cases of maximum obliquity, minimum and maximum tilt and minimum rotation gender has been noted to affect the results. Additionally, for maximum obliquity a statistically significant interaction (PERT*GENDER) was observed between gender and the presence of perturbation (PERT factor).

Obliquity min
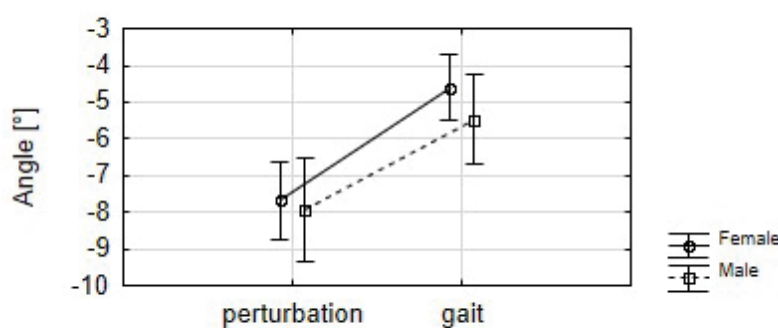

Tilt min
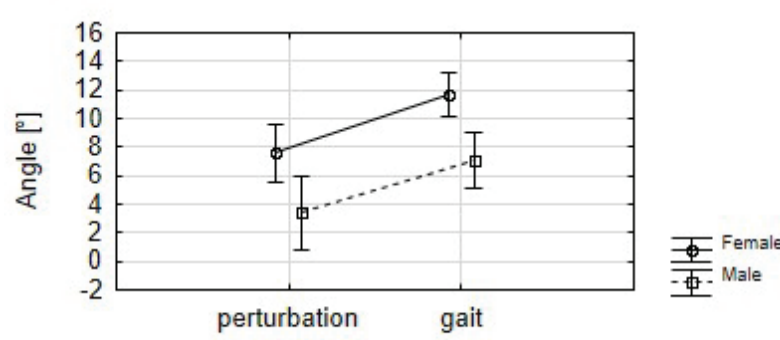

Rotation min
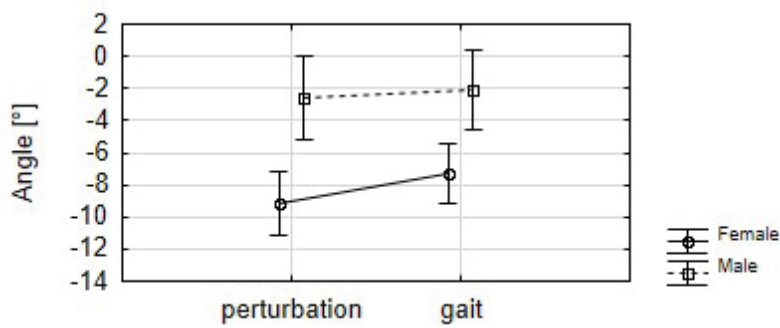

For females, the difference between gait and perturbation was greater compared to males (Fig. 2). On the other hand, a statistically significant difference between results for men and women was observed only for perturbation (Tab. 3). For perturbation, the maximum obliquity was greater compared to gait.

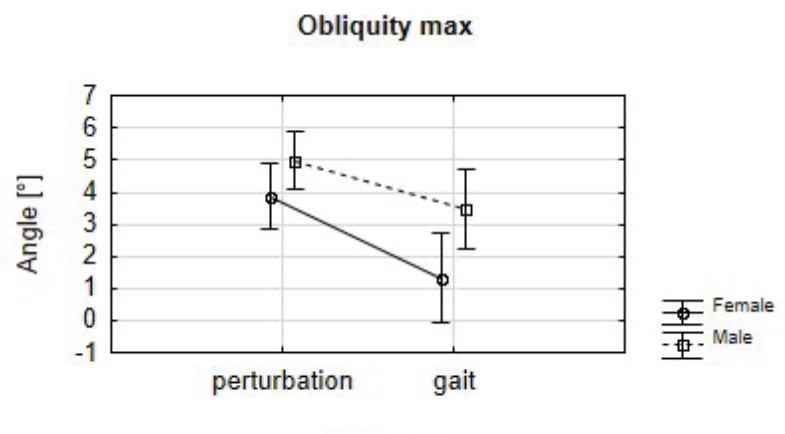

Tilt max

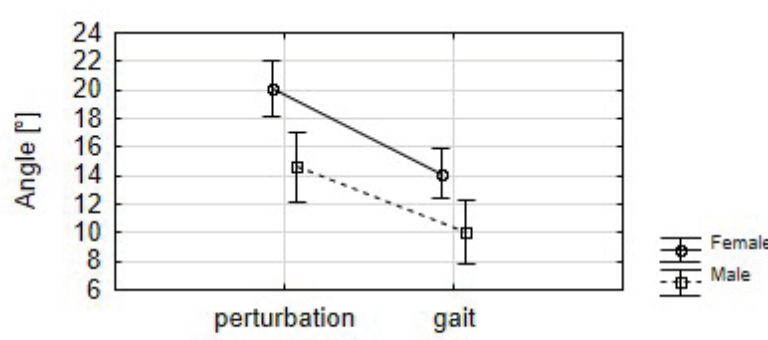

Rotation max

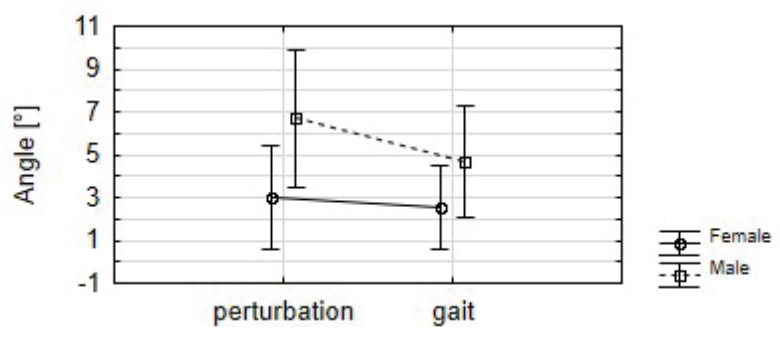

Fig. 2. Mean and $95 \%$ confidence intervals for variables describing pelvis kinematics for gait and perturbation for males and females separately

Tab. 3. p-value for post hoc test of selected parameters

\begin{tabular}{ccccc}
\hline & Gait vs perturbation (F) & Gait vs perturbation (M) & Gait (F) vs gait (M) & $\begin{array}{c}\text { perturbation }(\mathrm{F}) \\
\text { vs perturbation }(\mathrm{M})\end{array}$ \\
\hline obliquity min & $<\mathbf{0 . 0 0 1}$ & $\mathbf{< 0 . 0 0 1}$ & 0.785 & 0.993 \\
obliquity max & $\mathbf{0 . 0 0 1}$ & $<\mathbf{0 . 0 0 1}$ & 0.27 & $\mathbf{0 . 0 1 6}$ \\
tilt min & $<\mathbf{0 . 0 0 1}$ & $<\mathbf{0 . 0 0 1}$ & $\mathbf{0 . 0 1 4}$ & $\mathbf{0 . 0 2 7}$ \\
tilt max & $<\mathbf{0 . 0 0 1}$ & $<\mathbf{0 . 0 0 1}$ & $\mathbf{0 . 0 4 0}$ & $\mathbf{0 . 0 0 3}$ \\
rotation min & $\mathbf{0 . 0 1 1}$ & 0.907 & $\mathbf{0 . 0 2 7}$ & $\mathbf{0 . 0 0 3}$ \\
rotation max & & & & \\
\hline
\end{tabular}

Statistically significant value with $\alpha=0.05$ are bold

$\mathrm{F}$ - females, $\mathrm{M}$ - males 
Both for males and females, meaningfully lower pelvis obliquity was observed for perturbation when compared to gait (Fig. 1, Tab. 3). Maximum and minimum pelvic tilt was greater for females for gait as well as perturbation. On the contrary, for both males and females, greater maximum pelvic tilt and lower minimum pelvic tilt were observed for perturbation in comparison with gait.

The minimum rotation was lower for women compared to men by $6^{\circ}$ and $5^{\circ}$, respectively for perturbation and gait. Additionally, for females,

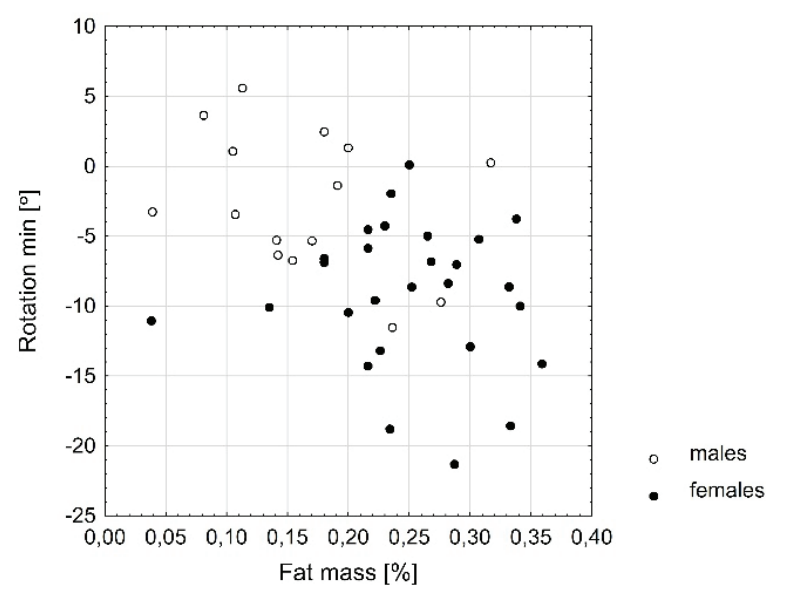

the minimum rotation was lower for perturbation compared to gait, which was not observed in males.

Statistically significant Pearson correlation coefficient was noted between minimum rotation and fat mass percentage (negative correlation) and muscle mass percentage (positive correlation), however, the analysis of the scatter plot made the authors question the results of the conducted analysis. It can be observed that males are characterized by higher muscle mass percentage and lower fat mass percentage while presenting lower right pelvis twist in comparison to females.

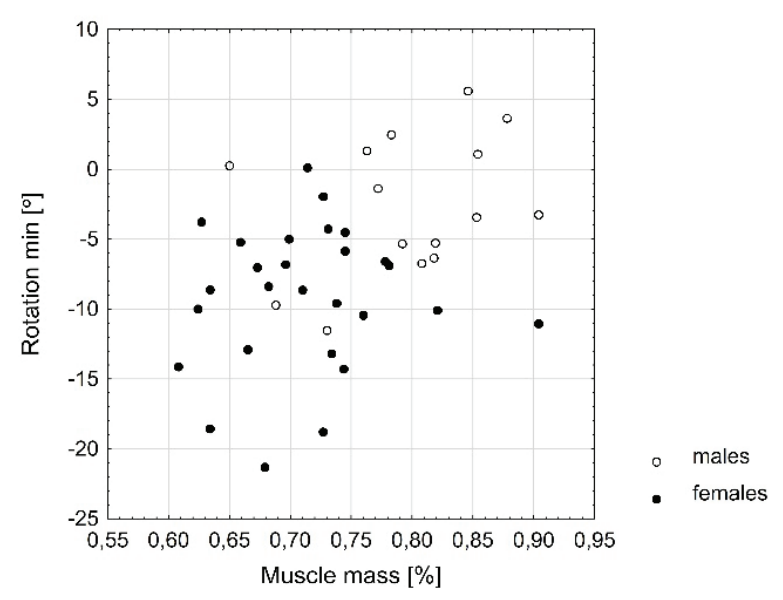

Fig. 3. Scatter plot showing the relationship between minimum rotation and fat mass percentage (on the left) and muscle mass percentage (on the right) with males and females marked separately

\section{Discussion}

Research concerning the body's response to perturbation concentrates mostly on the anteroposterior direction since that is the plane in which people usually sway [14]. In the current study, the pelvis reaction to perturbation was observed in all three analyzed planes, even though the perturbation was applied only in the sagittal plane. This suggests that pelvis reaction to perturbation is a three-dimensional problem. A similar observation was presented by Vlutters [15], who found the body's response to perturbation also in the planes perpendicular to the direction of applied perturbation.

Greater maximum pelvic tilt and lower minimum pelvic tilt for perturbation compared to normal gait indicates that shortly after gait perturbation people tend to lean back compared to their position during gait, whereupon they quickly compensated this movement by tilting the pelvis more forward.
A similar mechanism can be observed for pelvic obliquity. Minimum pelvis obliquity was lower for perturbation compared to gait; contrarily maximum pelvic obliquity was greater for perturbation compared to gait.

Kinematic parameters describing pelvis motion in sagittal and coronal planes were described by Vlutters [15]. Yet, even though he observed similarities in pelvis tilt comparable to the current study, he concentrated mostly on kinematics and kinetics of lower extremities and the deeper analysis of pelvis motion was not undertaken.

The work of the pelvis while perturbation was brought up in the work of Shumway-Cook [14], who considered the role of the hip joint. Body balance strategies presented in her research refer to the standing position, yet the author states that they also pertain to gait perturbations such as a trip or slip. Body balance strategy, which is in agreement with observed pelvis kinematics, is the hip joint strategy in reaction to the perturbation in the sagittal 
plane. People subjected to perturbation flex their hip joints and at the same time lean the trunk and the pelvis forward. Additionally, the author claims that because of a very low range of motion in the coronal plane for knee and ankle joints, it is in fact the hip that is responsible for compensatory body position and pelvis motion.

The importance of the role of the pelvis in controlling posture after becoming unbalanced can be demonstrated by studies showing significant differences in the muscular stimulation pattern in people who have pain at the level of the lumbar spine $[16,17]$ compared to people without it.

Moreover, pain in the spine is more frequent in women, what additionally may increase the risk of falling for this group [18]. Hence, the inclusion of gender in the analysis seems to be justified.

An interesting founding in the current study is a greater right pelvic twist for women compared to men. No mentions of the role of the transversal plane in body balance control was found in the literature.

Differences in pelvic response to gait perturbation with respect to the subject's gender were observed for each of the analyzed planes. This finding is interesting because, as stated earlier, a higher percentage of falls is observed in women $[2,4]$. The influence of gender on the results of the kinematic analysis was observed both in gait and during tripping.

As indicated in the introduction women exhibit greater pelvis tilt $[11,12]$ and greater range of motion in the coronal plane while walking compared to men [12]. In current study the greater pelvic tilt for women observed in gait is in accordance with the literature $[11,12]$ and occurs during gait perturbation as well. However, greater pelvic mobility in the coronal plane for gait was not observed in this research. Observed in current study differences in pelvis rotation between males and females were not found in the literature. Yet, increased right pelvis twist for women, for both gait and tripping, may indicate worse pelvic stability. Cases of women's poorer reaction to various types of balance disturbances such as standing position perturbation or rapid single leg lift have been describedin the literature [18-20].

The analysis of the relationship between the kinematic parameters of the pelvis and the percentage of muscle and fat tissue indicates that to reliably calculate the Pearson correlation coefficient, women and men ought to be analyzed separately.
Such analysis with the current study group, probably due to its size, does not show the observed in current study relationship for either gender.

Though, after analyzing the graph shown in Fig. 3, it can be assumed that the greater right pelvis twist observed for women, in addition to anatomical differences in the structure of the pelvis, may also be associated with the smaller percentage of muscle tissue combined with the larger percentage of body fat recorded in this group. There is a need for further research concerning this relationship, aimed to find the answer to the question whether pelvic stabilization training could reduce the risk of falling.

Dubey [21] describes the beneficial effects of training aimed at stabilizing the pelvis in people who have suffered a stroke, compared to the traditional physiotherapy. The author observed a significant improvement in, among others, trunk and lower limbs control and muscle strength in the hip area.

Among the main limitations of this work is the study group's uneven division, gender-wise. There were fewer men than women, which could have affected the results of the conducted statistical tests.

Moreover, the testing was performed in laboratory conditions, however, the use of a special two-belts treadmill driven by the participants and including virtual reality component allowed for the conditions to be as close to the natural environment as possible [22]. The creators of the system claim that the Motek system allows to simulate trips and slips in close to real conditions, nevertheless the actual walking takes place on a treadmill and not on a natural surface.

The issues described in the discussion relate to induced gait perturbation, simulating tripping, yet further research is needed to determine whether the presented conclusions would be true for other types of uncontrolled falls. What it more, not many papers were found that would allow a direct comparison of the obtained test results to other findings.

\section{Conclusion}

A significant pelvic response to gait perturbation was observed. Based on the results, it can be assumed that differences in the anatomy and biomechanics of the pelvis may be associated with greater susceptibility to uncontrolled falls during normal gait for women compared to men. 


\section{References}

1. Hausdorff JM, Rios DA, Edelberg HK. Gait variability and fall risk in community-living older adults: A 1-year prospective study. Arch Phys Med Rehabil. 2001;82(8):1050-6.

2. Stevens JA, Corso PS, Finkelstein EA, Miller TR. The costs of fatal and non-fatal falls among older adults. Inj Prev. 2006;12(5):290-5.

3. Campbell AJ, Reinken J, Allan BC, Martinez GS. Falls in old age: a study of frequency and related clinical factors. Age Ageing. 1981;10(4):264-70.

4. Dimitrova N, Zamudio JR, Jong RM, Soukup D, Resnick R, Sarma K, Ward AJ, Raj A, Lee J, Sharp PA, Jacks T. Falls Among Adults. Am J Prev Med. 2010;32(7):736-40.

5. Chang W, Leclercq S, Lockhart TE, Haslam R. State of science : occupational slips, trips and falls on the same level. Ergonomics [Internet]. 2016;59(7):1-23. Available from: http://dx.doi.org/10.1080/00140139.2016.1157214

6. Piercha K, Niemczyk K. zapobiegania Falls - epidemiology , risk factors and prevention strategy. 2013;2:1-10.

7. Gabell A, Simons MA, Nayak ULS. Falls in the healthy elderly: Predisposing causes. Ergonomics. 1985;28(7):965-75.

8. Sheldon JH. On The Natural History of Falls in Old Age. Br Med J. 1960;2(5214):1685-90.

9. Sung PS, Danial P. Gender difference of shoulder-pelvic kinematic integration for trunk rotation directions in healthy older adults. Clin Biomech [Internet]. 2017;50(May):56-62. Available from: https://doi.org/10.1016/j.clinbiomech.2017.09.014

10. Tejszerska D, Świtoński E, Gzik M. Biomechanika narządu ruchu człowieka. Instytut Technologii Eksploatacji; 2011.

11. Cho SH, Park JM, Kwon OY. Gender differences in three dimensional gait analysis data from 98 healthy Korean adults. Clin Biomech. 2004;19(2):145-52.

12. Stansfield B, Hawkins K, Adams S, Bhatt H. A mixed linear modelling characterisation of gender and speed related changes in spatiotemporal and kinematic characteristics of gait across a wide speed range in healthy adults. Med Eng Phys [Internet]. 2018;60:94-102. Available from: https://doi.org/10.1016/j.medengphy.2018.07.015

13. Van Den Bogert AJ, Geijtenbeek T, Even-Zohar O, Steenbrink F, Hardin EC. A real-time system for biomechanical analysis of human movement and muscle function. Med Biol Eng Comput. 2013;51(10):1069-77.

14. Shumway-Cook A, Woollacott MH. Motor control: translating research into clinical practice. Lippincott Williams \& Wilkins; 2007.

15. Vlutters M, van Asseldonk EHF, van der Kooij H. Lower extremity joint-level responses to pelvis perturbation during human walking. Sci Rep [Internet]. 2018;8(1):1-12. Available from: http://dx.doi.org/10.1038/s41598-018-32839-8

16. Sperry MM, Phillips ATM, Mcgregor AH. Lower back pain and healthy subjects exhibit distinct lower limb perturbation response strategies: A preliminary study. J Back Musculoskelet Rehabil. 2019;32(1):27-35.

17. Jacobs J V., Henry SM, Jones SL, Hitt JR, Bunn JY. A history of low back pain associates with altered electromyographic activation patterns in response to perturbations of standing balance. J Neurophysiol. 2011;106(5):2506-14.

18. Bussey MD, Castro MP de, Aldabe D, Shemmell J. Sex differences in anticipatory postural adjustments during rapid single leg lift. Hum Mov Sci [Internet]. 2018;57(May 2017):417-25. Available from: https://doi.org/10.1016/j.humov.2017.10.003

19. Sung PS, Park HS. Gender differences in ground reaction force following perturbations in subjects with low back pain. Gait Posture. 2009;29(2):290-5.

20. Miller EM, Slota GP, Agnew MJ, Madigan ML. Females exhibit shorter paraspinal reflex latencies than males in response to sudden trunk flexion perturbations. Clin Biomech. 2010;25(6):541-5.

21. Dubey L, Karthikbabu S, Mohan D. Effects of Pelvic Stability Training on Movement Control, Hip Muscles Strength, Walking Speed and Daily Activities after Stroke: A Randomized Controlled Trial. Ann Neurosci. 2018;25(2):80-9. 